\title{
Broad-band multicolour observations of the symbiotic binary $Z$ And during quiescence and its activity at the end of $2002^{\star}$
}

\author{
N. A. Tomov ${ }^{1}$, M. T. Tomova ${ }^{1}$, and O. G. Taranova ${ }^{2}$ \\ ${ }^{1}$ Institute of Astronomy, National Astronomical Observatory Rozhen, PO Box 136, 4700 Smolyan, Bulgaria \\ e-mail: tomov@astro.bas.bg \\ 2 Sternberg Astronomical Institute, Moscow State University, Universitetski pr. 13, Moscow, 119899 Russia
}

Received 9 April 2004 / Accepted 28 August 2004

\begin{abstract}
Broad-band $U B V R J H K L M$ photometry of the classical symbiotic binary system Z And obtained at the time of the light maximum of its small-amplitude brightening at the end of 2002 as well as at four epochs before and after this brightening is analysed. The parameters of the symbiotic nebula were obtained from its spectral energy distribution at the epochs of the quiescent state of the system using the energy distribution of the stellar components derived in a previous investigation. The continuum analysis based on the data during the active phase shows that the energy distribution of the compact secondary was changed very little - it remained a hot object emitting mostly in the UV, as in the quiescent state. On the basis of this result the brightening is supposed to be due to a slow expansion to about two times its quiescent size. The possibility of hydrogen burning at the surface of the secondary during quiescence is discussed.
\end{abstract}

Key words. stars: binaries: symbiotic - stars: activity - stars: individual: Z And - techniques: photometric

\section{Introduction}

Symbiotic stars are a group of eruptive binary systems whose hot compact component accretes matter from the atmosphere of its cool luminous companion. In some objects of this group accretion leads to an expansion of the compact component and a growth of the visual flux. Sometimes this growth is due to continuum energy redistribution caused by ejection of an optically thick shell and in other cases to increased emission of the circumbinary nebula as a result of a growth of the Lyman flux of the compact object.

The Z And system consists of a normal cool giant of spectral type M 4.5 (Mürset \& Schmid 1999), a hot compact component with a temperature higher than $10^{5} \mathrm{~K}$ (Fernandez-Castro et al. 1988) and an extended circumbinary nebula formed by the wind of the giant and partly photoionized by the hot component. The orbital period of this binary is $758^{\mathrm{d}} .8$ derived from both photometric (Formiggini \& Leibowitz 1994) and radial velocity (Mikolajewska \& Kenyon 1996) data.

The behaviour of its visual light is determined from an alternance of quiescent and eruptive phases. Several phases of activity (after 1915, 1939, 1960, 1984 and 2000) of Z And

* Based on observations collected at the Crimean Station of the Sternberg Astronomical Institute, Ukraine and the National Astronomical Observatory Rozhen, Bulgaria. were observed up to now, characterized by repeated brightenings with amplitudes up to $2-3 \mathrm{mag}$. The results of the observations during almost all of the active phases show energy redistribution in the continuum of the hot component caused by expansion of an optically thick shell (Swings \& Struve 1941; Boyarchuk 1967; Fernandez-Castro et al. 1995; Tomov et al. 2003a, hereafter Paper I).

The last major brightening of $\mathrm{Z}$ And began at the end of August 2000, the optical light reached its maximum in December and gradually decreased to its typical quiescent value untill the summer of 2002 (Skopal et al. 2000, 2002; Skopal 2003). In August 2002 there was not any indication of activity of the system and the light was in a deep minimum determined by an eclipse of the hot component (Skopal 2003). After that time the light increased again and reached its maximum in November, but the $U B V$ amplitudes were not great $\sim 1^{\mathrm{m}}$. At the same time, however, the IR emission was heavily enhanced - to the level close to that in December 2000. The star underwent an outburst again, but it was different to the major outbursts when the visual emission of the hot companion predominates over the other components (for example the 2000-2002 outburst) in its typical energy distribution - a weak $U B V$ emission and relatively strong JHKLM one. This paper is devoted to an analysis of the continuum energy emitted in these regions of the spectrum of $\mathrm{Z}$ And during its outburst at 
Table 1. The collected multicolour photometry.

\begin{tabular}{|c|c|c|c|c|c|c|c|c|c|c|c|}
\hline Date & $\begin{array}{l}\text { JD- } \\
2452000\end{array}$ & Phase & $U$ & $B$ & V & $R^{a}$ & $J$ & $H$ & $K$ & $L$ & $M$ \\
\hline 2002 Aug. 19 & 506.451 & 0.960 & 11.41 & 11.79 & 10.63 & 9.42 & 6.22 & 5.28 & 4.95 & 4.77 & 5.06 \\
\hline Quiescent & & & \pm 0.02 & \pm 0.02 & \pm 0.02 & \pm 0.02 & \pm 0.01 & \pm 0.01 & \pm 0.01 & \pm 0.02 & \pm 0.04 \\
\hline 2002 Nov. 12 & 591.308 & 0.080 & 10.10 & 10.76 & 9.85 & 8.80 & $6.10^{b}$ & $5.21^{b}$ & $4.88^{b}$ & 4.62 & 4.92 \\
\hline Active & & & \pm 0.02 & \pm 0.02 & \pm 0.02 & \pm 0.02 & & & & \pm 0.01 & \pm 0.02 \\
\hline 2003 Aug. 04 & 855.525 & 0.428 & 10.72 & $11.50^{c}$ & $10.42^{c}$ & $9.14^{c}$ & $6.25^{b}$ & 5.35 & 5.00 & 4.77 & 5.02 \\
\hline Quiescent & & & \pm 0.02 & & & & & \pm 0.01 & \pm 0.01 & \pm 0.01 & \pm 0.06 \\
\hline 2003 Aug. 17 & 869.400 & 0.447 & 10.70 & $11.52^{c}$ & $10.45^{c}$ & $9.15^{c}$ & $6.26^{b}$ & 5.35 & $4.99^{b}$ & 4.81 & 5.08 \\
\hline Quiescent & & & \pm 0.02 & & & & & \pm 0.01 & & \pm 0.01 & \pm 0.08 \\
\hline 2003 Oct. 06 & 919.294 & 0.512 & 10.65 & 11.50 & 10.47 & $9.15^{c}$ & 6.24 & $5.33^{b}$ & 4.99 & 4.77 & 5.01 \\
\hline Quiescent & & & \pm 0.03 & \pm 0.02 & \pm 0.03 & & \pm 0.01 & & \pm 0.01 & \pm 0.01 & \pm 0.04 \\
\hline
\end{tabular}

${ }^{a}$ Data of Skopal (2003) and Skopal et al. (2004).

${ }^{b}$ The inner uncertainty is of about 0.01 .

${ }^{c}$ The inner uncertainty is 0.005 .

the end of 2002. We continue our previous investigation described in Paper I. The aim is to obtain some parameters of the components, which would allow us to conclude about their behaviour during the outburst. When our analysis was carried out we were not successful in obtaining UV data to derive the parameters of the hot component. Thus, we tried to indirectly obtain an overall picture for its bechaviour using the data of the nebular emission and supposing that it is the ionizing source.

\section{Observations and reduction}

Broad-band JHKLM photometry of $\mathrm{Z}$ And was obtained on 29 Aug. 2002 (JD 2452516.437), 11 Dec. 2002 (JD 2452620.262), 22 July 2003 (JD 2452842.543), 15 Aug. 2003 (JD 2452866.563 ) and 13 Oct. 2003 (JD 2452926.360 ) with the InSb photometer attached to the $125 \mathrm{~cm}$ telescope of the Crimean Station of the Sternberg Astronomical Institute. Broad-band $U B V$ photometry was obtained on 21 July 2002 (JD 2452447.490 ), 12 Nov. 2002 (JD 2452591.308 ) and 6 Oct. 2003 (JD 2452 919.294) with the single channel photoelectric photometer, attached to the $60 \mathrm{~cm}$ telescope of the National Astronomical Observatory Rozhen. The comparison stars used were described in Paper I.

The collection of the data taken in eight photometric bands provides the possibility to analyse the light in a broad spectral region. Simultaneously, UBVR photometric observations by Skopal et al. (2004) were performed. We combined our IR photometry from 29 Aug. 2002 with his data of 19 Aug. since both sets of data have typical quiescent values and we can suppose that the system was in quiescence on 19 Aug. Knowing the quiescent continuum emission of the cool giant and the secondary stellar component, we can obtain the emission of the circumbinary nebula and calculate its parameters (see the next section) at that epoch.

After the time of the orbital minimum the light increased for two reasons - an increase of the nonocculted part of the nebula and a progress of activity. During active phase we do not know the continuum of the secondary component and moreover, it would not be correct to determine it on the basis of visual and IR data only, since this star is a hot object emitting mainly in the UV region (seen Sect. 4). For this reason we cannot analyse the continuum of the system at the epoch of our IR photometry.

The light curves of Skopal (2003) and Skopal et al. (2004) show that our $U B V$ estimate of November 2002 was obtained at the maximum of the light during the outburst at the end of the year 2002. For our considerations we used average $U B V$ magnitudes from the photometry of Skopal and our data at that time. We proceeded in the same way with the photometry from October 2003. Our JHKLM estimates from 22 July and 15 Aug. 2003 were combined with the $U B V$ estimates from 4 Aug. and 17 Aug. 2003 of Skopal et al. (2004). As a result we collected UBVJHKLM estimates (Table 1), derived at five epochs. Four of them are related to the low state of the light when the system reached its typical quiescent magnitudes and the other one to a phase of activity when the light had a maximal value (Fig. 1). Since the IR estimates were less changed they were related to the times of obtaining the $U B V$ magnitudes. We also used $R$ photometric data from the light curve of Skopal (2003) at the epoch of our observation from November 2002 and from the work of Skopal et al. (2004) for the other four epochs as well.

The stellar magnitudes were converted into continuum fluxes using the calibration from the book of Mihailov (1973, Table 2). The $U$ flux was corrected for the energy distribution of $Z$ And in the region of the Balmer jump in the same way as for the quiescent photometric data in Paper I. Our spectra taken in November 2002 at the light maximum show the same height of the $\mathrm{H} \gamma$ line above the continuum level as in the quiescent state of the system. Based on these data we suppose that the Balmer jump during this active phase is similar to in quiescence although the Balmer decrement possibly changes. Thus, for the photometric data from the active phase we used the same correction as for the quiescent data. 


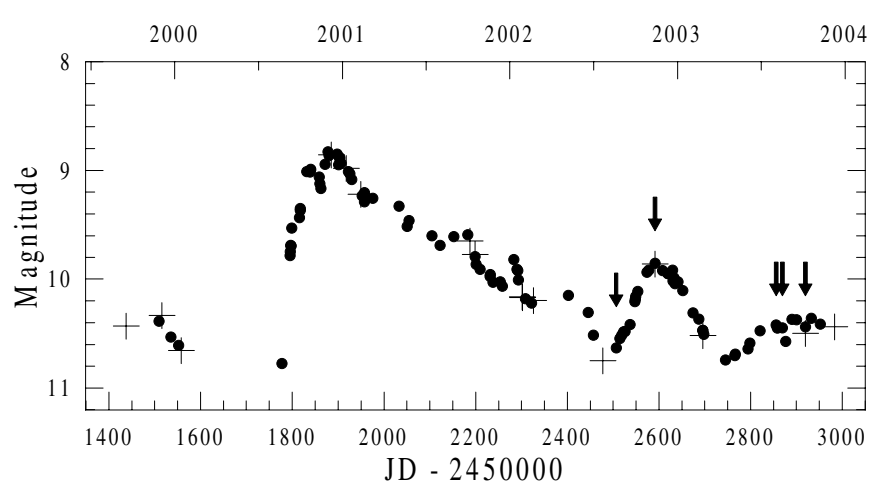

Fig. 1. The $V$ light curve of $\mathrm{Z}$ And during its phase of activity after the summer of 2000. The dots indicate the data of Skopal et al. (2002, 2004) and the crosses our unpublished data. The arrows indicate the epochs during which we obtained our multicolour UBVJHKLM data.

The $U B V$ fluxes of $\mathrm{Z}$ And were corrected for its strong emission lines in the same way as the quiescent data in Paper I, again because of the fact that the heights of these lines during the active phase were practically the same as those in the quiescence.

All fluxes were corrected for the interstellar reddening. We used the value $E(B-V)=0.30$ and proceeded according to the approaches of Seaton (1979) and Johnson (1966) as in Paper I.

We used also the ephemeris Min(vis) = JD $2442666^{\mathrm{d}}+$ $758.8 \times \mathrm{E}$, where the orbital period is based on both photometric and spectral data and the epoch of the orbital photometric minimum coincides with that of the spectral conjunction (Formiggini \& Leibowitz 1994; Mikolajewska \& Kenyon 1996; Fekel et al 2000).

\section{Analysis of the energy distribution}

As in Paper I the main aim of our study is to obtain some parameters of the hot stellar component and the circumbinary nebula during the active phase. These parameters can be determined from their continuum emission. It turned out, however, that the hot component has radiated comparatively little in the region of the photometric bands used by us and mainly at the shorter wavelengths. Thus, its parameters were determined also by means of one supposition about the physical conditions in the nebula in addition to the analysis of the observed continuum. To realize this analysis during an active phase we also need to know the emission of the cool giant in the system which has a basic contribution in its JHKLM emission.

An additional task of our study is to derive the quiescent parameters of the nebula at the times of our observation.

\subsection{The quiescent phase}

The $\mathrm{Z}$ And system had its typical quiescent UBVRJHKLM magnitudes at the times 19 Aug. 2002, 4 Aug., 17 Aug. and 6 Oct. 2003 (Table 1) and thus we consider that at those times it was in the quiescent state. The fluxes and the parameters of its cool giant were calculated from the data taken on 15 Sep. 1999 (Paper I). The IR magnitudes obtained at that time are very similar to the arithmetical mean values of the data taken on 15 Sep. 1999, 19 Aug. 2002, 4 Aug., 17 Aug. and 6 Oct. 2003. For this reason we will use the fluxes and the parameters of the giant obtained from the photometric data of Z And of 15 Sep. 1999. The effective temperature and the radius amount to $3400 \pm 50 \mathrm{~K}$ and $85 \pm 4 R_{\odot}$ at a distance to the system of $1.12 \mathrm{kpc}$ (Fernandez-Castro et al. 1988, 1995) and the fluxes are listed in Table 2.

The quiescent blackbody fluxes (Table 2, Fig. 2) and parameters (Table 3 ) of the hot component of the system were also determined in Paper I with use of UV data from the works of Nussbaumer \& Vogel (1989) and Fernandez-Castro et al. (1988, 1995).

The fluxes of the circumbinary nebula were obtained after subtraction of the stellar fluxes from the observed ones. The electron temperature and the emission measure result from fitting these fluxes with a nebular continuum. We applied the same procedure as in Paper I, adopting that doubly ionized helium is dominant in the nebula during the quiescent state and its emission is mostly continuum emission of hydrogen and ionized helium. The results are represented in Table 3. The uncertainties are determined from those of the observational data. The quiescent nebular fluxes in Table 2 and Fig. 2 are related to the corresponding parameters in Table 3.

\subsection{The active phase}

The most typical feature of the photometric behaviour of Z And at the end of the year 2002 was its strong IR emission whose intensity was close to that at the time of the light maximum in December 2000 during its major brightening. On the other hand the $U B V$ emission increased little compared to that brightening. When analyzing our data from November 2002 we proceeded in the following way. Initially we subtracted the fluxes of the cool giant from the observed fluxes. It turned out that the rest of the emission is fitted very well by a nebular continuum in the region of the RJHK bands. This fitting, however, suggests that additional emission of a third component is present in the $U B V$ region as well (Table 2).

To determine the parameters of the nebular emission it is necessary to know the dominant ionization state of helium in the surrounding nebula of the system. As in our previous investigation (Paper I) we calculated the ratio of the emission measures of the neutral and ionized helium using the fluxes of the He I 4471 and He II 4686 lines, which, however, this time were based on high resolution spectral data taken simultaneously with our photometry in November 2002. It was obtained that doubly ionized helium is again dominant and we assumed that the nebular continuum is formed by the emission of hydrogen and ionized helium.

To make the continuum fit it is necessary to have the quantities $\gamma_{v}$ related to the emission coefficients of these elements, determined by recombinations and free-free transitions. In Paper I we noted that the system $\mathrm{Z}$ And in its quiescence has an apparent continuum which on the long wavelength-side of the Balmer limit has the same flux as on the short wavelength-side because of the blending of the Balmer emission lines with high numbers. During the major brightening, however, the width of 
Table 2. The continuum fluxes of the system's components in units $10^{-12} \mathrm{erg} \mathrm{cm}^{-2} \mathrm{~s}^{-1} \AA^{-1}$. The fluxes of the giant and the quiescent fluxes of the hot component are according to Paper I. The uncertainties of the observed values are in the same units.

\begin{tabular}{|c|c|c|c|c|c|c|c|c|c|c|}
\hline Date & $\mathrm{SC}^{a}$ & $U$ & $B$ & $V$ & $R$ & $J$ & $H$ & $K$ & $L$ & $M$ \\
\hline 2002 Aug. 19 & Cool & 0.020 & 0.160 & 0.376 & 0.710 & 1.343 & 0.856 & 0.439 & 0.113 & 0.034 \\
\hline \multirow[t]{6}{*}{ Quiescent } & Hot & 0.030 & 0.014 & & & & & & & \\
\hline & Neb. & 0.560 & 0.162 & 0.142 & 0.108 & 0.031 & 0.018 & 0.011 & 0.004 & \\
\hline & $\mathrm{TF}$ & 0.580 & 0.322 & 0.518 & 0.818 & 1.374 & 0.874 & 0.450 & 0.117 & 0.034 \\
\hline & OF & 0.569 & 0.391 & 0.520 & 0.617 & 1.359 & 0.878 & 0.440 & 0.104 & 0.021 \\
\hline & & \pm 0.009 & \pm 0.007 & \pm 0.008 & \pm 0.012 & \pm 0.012 & \pm 0.009 & \pm 0.004 & \pm 0.002 & \pm 0.001 \\
\hline & $r$ & 2 & -18 & 0 & 32 & 1 & 0 & 2 & 12 & 62 \\
\hline 2002 Nov. 12 & Cool & 0.020 & 0.160 & 0.376 & 0.710 & 1.343 & 0.856 & 0.439 & 0.113 & 0.034 \\
\hline \multirow[t]{6}{*}{ Active } & Hot & 0.107 & 0.052 & 0.022 & & & & & & \\
\hline & Neb. & 1.639 & 0.730 & 0.640 & 0.485 & 0.138 & 0.079 & 0.050 & 0.020 & \\
\hline & $\mathrm{TF}$ & 1.766 & 0.942 & 1.038 & 1.195 & 1.481 & 0.935 & 0.489 & 0.133 & 0.034 \\
\hline & OF & 1.902 & 1.010 & 1.068 & 1.093 & 1.518 & 0.936 & 0.470 & 0.119 & 0.024 \\
\hline & & \pm 0.033 & \pm 0.021 & \pm 0.021 & \pm 0.021 & \pm 0.001 & \pm 0.001 & \pm 0.001 & \pm 0.001 & \pm 0.001 \\
\hline & $r$ & -7 & -7 & -3 & 9 & -2 & 0 & 4 & 12 & 42 \\
\hline 2003 Aug. 04 & Cool & 0.020 & 0.160 & 0.376 & 0.710 & 1.343 & 0.856 & 0.439 & 0.113 & 0.034 \\
\hline \multirow[t]{6}{*}{ Quiescent } & Hot & 0.030 & 0.014 & & & & & & & \\
\hline & Neb. & 1.055 & 0.306 & 0.268 & 0.203 & 0.058 & 0.033 & 0.021 & 0.008 & \\
\hline & $\mathrm{TF}$ & 1.105 & 0.480 & 0.644 & 0.913 & 1.401 & 0.889 & 0.460 & 0.121 & 0.034 \\
\hline & OF & 1.075 & 0.511 & 0.631 & 0.798 & 1.323 & 0.822 & 0.420 & 0.104 & 0.022 \\
\hline & & \pm 0.020 & \pm 0.003 & \pm 0.003 & \pm 0.003 & \pm 0.012 & \pm 0.007 & \pm 0.004 & \pm 0.001 & \pm 0.001 \\
\hline & $r$ & 3 & -6 & 2 & 14 & 6 & 8 & 10 & 16 & 54 \\
\hline 2003 Aug. 17 & Cool & 0.020 & 0.160 & 0.376 & 0.710 & 1.343 & 0.856 & 0.439 & 0.113 & 0.034 \\
\hline \multirow[t]{6}{*}{ Quiescent } & Hot & 0.030 & 0.014 & & & & & & & \\
\hline & Neb. & 1.033 & 0.299 & 0.262 & 0.199 & 0.057 & 0.032 & 0.020 & 0.008 & \\
\hline & $\mathrm{TF}$ & 1.083 & 0.473 & 0.638 & 0.909 & 1.400 & 0.888 & 0.459 & 0.121 & 0.034 \\
\hline & OF & 1.095 & 0.501 & 0.614 & 0.791 & 1.311 & 0.822 & 0.424 & 0.100 & 0.021 \\
\hline & & \pm 0.020 & \pm 0.002 & \pm 0.003 & \pm 0.004 & \pm 0.012 & \pm 0.007 & \pm 0.004 & \pm 0.001 & \pm 0.002 \\
\hline & $r$ & -1 & -6 & 4 & 15 & 7 & 8 & 8 & 21 & 62 \\
\hline 2003 Oct. 06 & Cool & 0.020 & 0.160 & 0.376 & 0.710 & 1.343 & 0.856 & 0.439 & 0.113 & 0.034 \\
\hline \multirow[t]{6}{*}{ Quiescent } & Hot & 0.030 & 0.014 & & & & & & & \\
\hline & Neb. & 1.055 & 0.306 & 0.268 & 0.203 & 0.058 & 0.033 & 0.021 & 0.008 & \\
\hline & $\mathrm{TF}$ & 1.105 & 0.480 & 0.644 & 0.913 & 1.401 & 0.889 & 0.460 & 0.121 & 0.034 \\
\hline & OF & 1.146 & 0.511 & 0.603 & 0.791 & 1.335 & 0.838 & 0.424 & 0.104 & 0.022 \\
\hline & & \pm 0.026 & \pm 0.010 & \pm 0.016 & \pm 0.004 & \pm 0.012 & \pm 0.001 & \pm 0.004 & \pm 0.001 & \pm 0.001 \\
\hline & $r$ & -4 & -6 & 7 & 15 & 5 & 6 & 8 & 16 & 54 \\
\hline
\end{tabular}

${ }^{a}$ The system's components, $\mathrm{TF}=\mathrm{Cool}+\mathrm{Hot}+$ Nebular: total flux; OF: observed flux; $r=(\mathrm{TF}-\mathrm{OF}) / \mathrm{OF}$ in $\%$.

the lines decreased, their blending changed and led to a decrease of the flux at $\lambda 3650 \AA$. In this case we used the arithmetical mean of the values of the hydrogen coefficient on both sides of the Balmer limit (Osterbrock 1974). We proceeded in the same way when analysing our data from November 2002.

The best fit at the wavelength positions of the UBVRJHKL bands for the nebular emission of the star during the active phase turned out to be continuum of gas with an electron temperature of $20000 \pm 1000 \mathrm{~K}$ and an emission measure of $(11.7 \pm 0.3) \times 10^{59}(d / 1.12 \mathrm{kpc})^{2} \mathrm{~cm}^{-3}$ (Fig. 2, Table 3). The nebular fluxes in Table 2 are related to these parameters. This result showed that the increase of the light of $\mathrm{Z}$ And at the time of its optical maximum was mainly due to the nebular emission and the energy distribution of its secondary stellar component was typical of a hot object radiating predominantly in the UV region, as in the quiescent state. 
Table 3. Parameters of the hot stellar and the nebular components based on the observations.

\begin{tabular}{llllll}
\hline \hline Date & $\begin{array}{l}\text { Component } \\
\text { of the system }\end{array}$ & $\begin{array}{l}T_{\text {eff }} \\
(\mathrm{K})\end{array}$ & $\begin{array}{l}R(d / 1.12 \mathrm{kpc}) \\
\left(R_{\odot}\right)\end{array}$ & $\begin{array}{l}T_{\mathrm{e}} \\
(\mathrm{K})\end{array}$ & $\begin{array}{l}n_{\mathrm{e}}^{2} V(d / 1.12 \mathrm{kpc})^{2} \\
\times 10^{59}\left(\mathrm{~cm}^{-3}\right)\end{array}$ \\
\hline $\begin{array}{l}\text { 2002 Aug. 19 } \\
\text { Quiescent }\end{array}$ & Not & $150000^{a}$ & $0.06^{a}$ & $20000 \pm 1000$ & $2.6 \pm 0.1$ \\
\hline 2002 Nov. 12 & Hot & $125000 \pm 3000$ & $0.13 \pm 0.01$ & & \\
Active & Nebular & & & $20000 \pm 1000$ & $11.7 \pm 0.3$ \\
\hline 2003 Aug. 04 & Hot & $150000^{a}$ & $0.06^{a}$ & & \\
Quiescent & Nebular & & & $20000 \pm 1000$ & $4.9 \pm 0.1$ \\
\hline $\begin{array}{l}\text { 2003 Aug. 17 } \\
\text { Quiescent }\end{array}$ & Hot Nebular & $150000^{a}$ & $0.06^{a}$ & & \\
\hline $\begin{array}{l}\text { 2003 Oct. 06 } \\
\text { Quiescent }\end{array}$ & Hot Nebular & $150000^{a}$ & $0.06^{a}$ & $20000 \pm 1000$ & $4.8 \pm 0.1$ \\
\hline
\end{tabular}

${ }^{a}$ Based on UV data (Nussbaumer \& Vogel 1989; Fernandez-Castro et al. 1988, 1995).

In this case it would not be correct to determine its parameters on the basis only of visual data. Thus, we obtained these parameters from both the $U B V$ fluxes and the companion's Lyman luminosity. It was necessary for this luminosity also to be estimated. Moreover we supposed that the gas medium in the nebula is in ionization equilibrium, i.e. the number of ionizing photons is equal to the number of recombinations. We did not have UV data giving us the possibility to obtain the number of ionizing photons from observations. The estimation of the Lyman luminosity of the companion is based on the following consideration.

The increase of the emission measure of the nebula (Table 3) indicates an increase of the number of recombinating ions by a factor of $2.5 \pm 0.2$ compared with the time of the quiescent state on 15 Sep. 1999 (Paper I) at the phase of the orbital maximum which requires an increase of the total Lyman luminosity of all ionizing sources in the system at least by the same factor. The basic source of ionizing emission is the hot component of the system. In the case when initially only a part of the nebula has been ionized and after the increase of the emission of the hot component - the whole one (except some small part behind the giant), the Lyman luminosity can have increased by a factor greater than that of the emission measure and some part of the photons can have left the nebula unabsorbed. In the quiescent state of the system $\mathrm{Z}$ And, its circumbinary nebula is only partly ionized (Fernandez-Castro et al. 1988; Mikolajewska \& Kenyon 1996; Schmid \& Schild 1997; Birriel et al. 1998). During its major outburst (2000-2002) the emission measure increased by a factor of 4.4 (Paper I). During the last outburst considered by us, the emission measure increased by a factor of 2.5 (Table 3 ). This means that some part of the nebula has probably remained a neutral region again and the increase of the Lyman luminosity has probably been by a factor practically equal to that of the increase of the emission measure.

In the case, when the basic source of ionization is the hot component, an additional ionization can be realized also by some other mechanism - for example wind collision. In such a case the increase of the Lyman luminosity of the hot companion is smaller than that of the emission measure. The luminosity of the shock region of the winds has a typical value of about $10 L_{\odot}$, which is small compared to the luminosity of the hot companion and cannot be taken into consideration.

Using the quiescent radius and effective temperature adopted in Paper I, we obtained a Lyman photon luminosity of $9.43 \times 10^{46}$ phot s$^{-1}$. We supposed that the Lyman luminosity has increased in the same ratio of $2.5 \pm 0.2$ as the emission measure of the nebula and in this case obtained $(2.36 \pm 0.19) \times 10^{47}$ phot s$^{-1}$. Using this value and the observed $U B V$ fluxes, for the radius and the effective temperature at the time of the optical maximum we derived $0.13 \pm 0.01 R_{\odot}$ and $125000 \pm 3000 \mathrm{~K}$. The uncertainties are determined from our observed fluxes. These parameters are given in Table 3 and the fluxes of the hot component (Table 2, Fig. 2) were calculated on their basis.

\section{Discussion}

The commonly accepted view is that hydrogen steady-state burning is realized at the surface of the secondary component of Z And in its quiescent state (Mikolajewska \& Kenyon 1992; Fernandez-Castro et al. 1995). This component is a high temperature compact object (Nussbaumer \& Vogel 1989). Its expansion could then be due to an accumulation of matter not included in the burning process as a result of the growth of the accretion rate. The expansion occurs at constant bolometric luminosity (Fujimoto 1982). The quiescent parameters used by us and the assumed increase of the Lyman luminosity by a factor of 2.5 lead to an increase of the bolometric luminosity of this hot object by a factor of about 2 , which however, makes the interpretation difficult for an expansion at constant bolometric luminosity. Though, we will use the relation between the velocity of the expansion and the accretion rate of an accreting white dwarf with hydrogen burning at its surface to obtain at 
least a most crude estimate of the increase of the accretion rate during the active phase. This velocity is given by

$$
\begin{aligned}
\frac{\mathrm{d} R}{\mathrm{~d} t} & =R \frac{\mathrm{d} \ln R}{\mathrm{~d} \ln \Delta M_{1}}\left(\frac{\dot{M}-\dot{M}_{\mathrm{RG}}}{\Delta M_{1}}\right) \\
& \approx 8 \mathrm{~m} \mathrm{~s}^{-1}\left(\frac{\dot{M}-\dot{M}_{\mathrm{RG}}}{10^{-6} M_{\odot} \mathrm{yr}^{-1}}\right)\left(\frac{\Delta M_{1}}{10^{-5} M_{\odot}}\right)^{-1}
\end{aligned}
$$

where $\mathrm{d} \ln R / \mathrm{d} \ln \Delta M_{1}=3.1 \sim 4.1$ at $R=1 R_{\odot}$ (Fujimoto 1982). $\dot{M}$ is the accretion rate necessary to trigger the expansion and $\Delta M_{1}$ is the mass of the accreted envelope. $\dot{M}_{\mathrm{RG}}$ is the upper limit of the region of the accretion rates, where the accretion rate is equal to the burning rate; when the accretion rate is greater than this limit the envelope expands as a result of accumulation of mass. Using this relation we can obtain an estimate of the mean accretion rate needed for expansion of the hot component of $\mathrm{Z}$ And for the time of the light increase from the mean velocity of the increase of the star's radius. The typical time of the growth of the light is $100^{\mathrm{d}}$ (Skopal 2003). In this case the mean velocity of the increase of the radius from $0.06 R_{\odot}$ to $0.13 R_{\odot}$ is obtained to be $5.6 \mathrm{~m} \mathrm{~s}^{-1}$. At a mass of the hot component of $0.6 M_{\odot}$ (Fernandez-Castro et al. 1988; Schmid \& Schild 1997) the mass of the accreted envelope for burning in thermal equilibrium is $5.01 \times 10^{-5} M_{\odot}$. The upper limit of the accretion rate is $1.77 \times 10^{-7} M_{\odot} \mathrm{yr}^{-1}$ (Fujimoto 1982). Then, using the relation 1 , we derived $3.2 \times 10^{-7} M_{\odot} \mathrm{yr}^{-1}$ for the accretion rate providing the expansion. According to Fernandez-Castro et al. (1988) the quiescent accretion rate of the hot component of the $\mathrm{Z}$ And system is equal to $4.5 \times 10^{-9} M_{\odot} \mathrm{yr}^{-1}$. In this case the ratio of the two values is about 70 .

One possible reason for the increase of the accretion rate was proposed by Bisikalo et al. (2002). They theoretically considered the evolution of the gas flow in the vicinity of the accretor of $\mathrm{Z}$ And and reached the conclusion that the accretion rate can strongly increase as a result of a change of the velocity of the cool giant wind. According to them there is a small range of velocities where the accretion rate increases by a factor of up to about 30. Our result for the increase of the accretion rate to its mean value during the outburst exceeds this theoretical prediction by a factor of about 2 .

We considered the possibility that the hot component expands because of accumulation of mass. Another mechanism is mass ejection. During the increase of the optical light a broad emission component of the high excitation He II 4686 line of $\mathrm{Z}$ And was observed, emitted probably in a region of stellar wind with high velocity. The width of this component at the level of the continuum corresponds to a velocity of the wind of about $1000 \mathrm{~km} \mathrm{~s}^{-1}$ (Tomov et al. 2004). It is possible that the radius of the star during the outburst can be determined from the level where the optical depth in the wind reaches unity in the continuum. This possibility, however, will be considered in detail after a comprehensive analysis of the emission line spectrum.

Sokoloski \& Bildsten (1999) obtained a 28 min oscillation of the $B$ light of $\mathrm{Z}$ And with an amplitude of 2-5 mmag and supposed that it is due to rotation of an accreting white dwarf

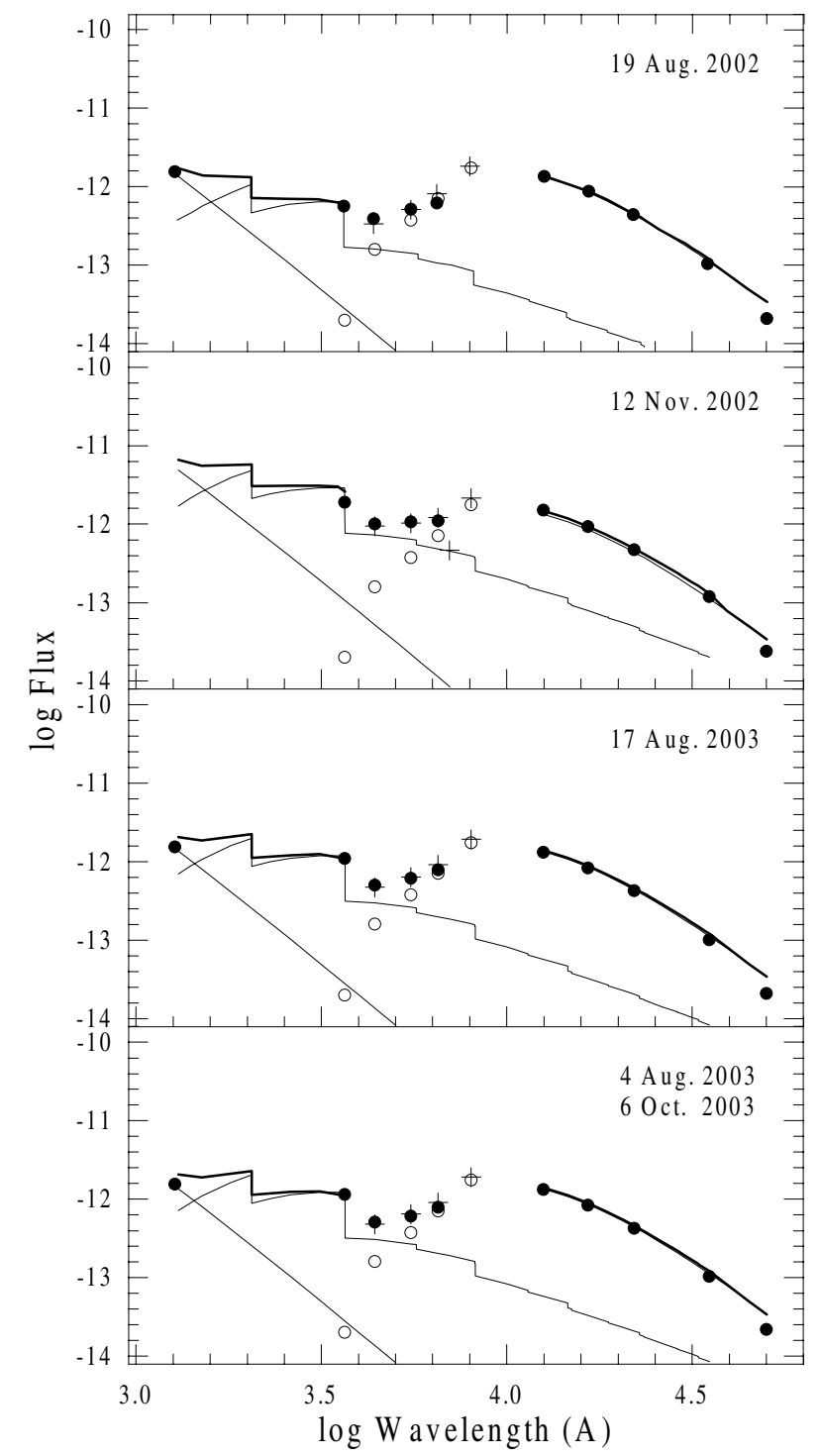

Fig. 2. The spectral energy distribution of $Z$ And during the times of our observations. The points indicate the observed fluxes. The thin lines represent the continua of the system's components. The continua of the giant and the hot component in quiescence are according to Paper I. The circles represent the UBVR fluxes of the giant according to Paper I. The thick line represents the resulting continuum. In the region of the $B V R$ photometric bands the resulting continuum is represented by crosses, placed only at their positions, since the giant does not radiate as a blackbody in this region and its continuum is not known at the other wavelengths. The quiescent UV flux of Fernandez-Castro et al. (1995) is also displayed. Flux units are erg cm $\mathrm{cm}^{-2} \mathrm{~s}^{-1}$.

with a strong enough magnetic field to channel the accretion flow onto the magnetic polar caps. At a distance equal to the radius of the magnetosphere the magnetic field pressure is comparable to the ram pressure of the accreting matter. The authors suppose that the period of the rotation $P$ is equal to the Kepler period on the boundary surface of the magnetosphere. In this case the magnetospheric radius is equal to the corotation radius

$R_{\mathrm{mag}}=R_{\mathrm{c}}=\left(\frac{G M_{\mathrm{WD}} P^{2}}{4 \pi^{2}}\right)^{1 / 3}$. 
We calculate the Keplerian velocity at a distance of $0.13 R_{\odot}$ equal to the radius at the time of the light maximum. This result shows that a white dwarf having such a radius and a mass of $0.6 M_{\odot}$ can rotate with a period of $28^{\mathrm{m}}$ since the velocity at that radius is smaller than the Keplerian velocity. Using the relation 2 we calculate also the magnetospheric radius. We obtained a value of $0.26 R_{\odot}$ greater than the radius of the white dwarf. This result means that the accretion must be driven by the magnetic field during the whole time of the outburst phase.

An other possibility for the physical conditions in the nebula during the outburst is related to absence of ionization equilibrium. In this case the basic ionizing source is not the hot companion, but rather some mechanism of collisional ionization. Such a mechanism was recently suggested by Kilpio et al. (2004). They have performed modeling of the structure of the gas flows in a binary system with parameters close to those of $Z$ And. The change of the velocity of the giant's wind leads to a change in the accretion regime. Moreover the hydrogen burning in the outer layers of the accreting white dwarf was taken into account by introducing a pressure jump at its surface. When the velocity of the wind increases, the presence of this jump leads to the appearance of shock waves in the area between the stellar components and to liberation of mechanical energy. This energy is spent on additional ionization. The change of the accretion rate can lead to change of the burning regime at the accretor. Its luminosity can increase and to contribute also to additional ionization of the nebula. In this case a combination of two ionizing sources is possible to present in the system. Their action can increase its light with a typical amplitude close to the observed ones.

Finally we compare the behaviour of the system Z And during its two optical brightenings: the first one having a large amplitude in the visual (the region $U B V$ ) and occuring in the period 2000-2002 and the second one with a small visual amplitude, at the end of 2002. During the major outburst the continuum energy distribution of the hot secondary underwent a pronounced change and its emission predominated in the $U B V$ region. The JHKLM fluxes also increased, but the nebular emission was the basic contributor to their growth. This behaviour was interpreted as an ejection of an optically thick shell with photospheric characteristics when the Lyman luminosity increased (Tomov et al. 2003b, Paper I). During the small-amplitude brightening the increase of the fluxes in the region $U B V R J H K L$ was mainly due to the nebular emission. The energy distribution of the secondary component changed very little, it remained a hot compact object as in the quiescent state of the system.

\section{Conclusions}

We present the results of broad-band UBVRJHKLM observations of the symbiotic binary $\mathrm{Z}$ And taken at five epochs. At four of them the system was in the quiescent state before and after its small-amplitude visual brightening at the end of 2002 . The fifth epoch is related to the maximum of the light during the brightening.

A continuum analysis was carried out and the emission of the hot and the nebular components of the system have been determined. The emission of the cool giant and the hot component in quiescence was known from previous investigations and their fluxes were subtracted from the quiescent observed fluxes. The difference obtained is related to the nebular emission and on this basis the electron temperature and the emission measure of the circumbinary nebula were determined.

The analysis of the data at the time of the light maximum showed that the RJHKL emission is formed from the continua of the cool giant and the circumbinary nebula. The $U B V$ emission, on the other hand, is formed of three parts - these two continua and a third one from the hot stellar component. The data show that during the outburst it was a compact object emitting mostly in UV, as in quiescence.

Two possibilities for the physical interpretation of the results of the analysis were considered. The first one is related to the case when the nebula is in ionization equilibrium as the hot companion is supposed to be the ionizing source. In this case the increase of the emission of the circumbinary nebula indicates an increase of the flux of ionizing photons. We assumed that the Lyman luminosity increases in the same ratio as the emission measure of the nebula. On the basis of the Lyman luminosity estimate and the $U B V$ fluxes we calculated the effective temperature and radius of the companion. The result shows that it expands to about two times its quiescent size. A commonly accepted view is that hydrogen burns in a steady state at the surface of the companion in quiescence. Our result, however, is inconsistent with this model since it proposes a growth of the bolometric luminosity. We suppose that the expansion is related to either an accumulation of matter as a result of an accretion rate increase or mass ejection. We also tried to obtain a crude estimate of the mean accretion rate during the active phase and the result was close to the theoretical prediction.

We considered the possibility of accretion on a fast rotating compact object with a radius equal to that obtained by us to be driven by a strong magnetic field as was suggested for the hot component of the system Z And by Sokoloski \& Bildsten (1999). It turned out that during the whole time of the outburst the radius remains smaller than the radius of the magnetosphere and accretion can be driven by the field.

The second possibility is that the nebula is not in ionization equilibrium during the time of activity. In this case the optical brightening could be interpreted with a mechanism of collisional ionization in the gas medium in addition to the radiative ionization by the hot component (Kilpio et al. 2004).

Acknowledgements. The authors thank the referee whose remarks contributed to improvement of the paper. N.T. is also indebted to Drs. D. Bisikalo and E. Kilpio for their helpful comments.

\section{References}

Birriel, J. J., Espey, B. R., \& Schulte-Ladbeck, R. E. 1998, ApJ, 507, L75

Bisikalo, D. V., Boyarchuk, A. A., Kilpio, E. Yu., \& Kuznetsov, O. A. 2002, ARep, 46, 1022

Boyarchuk, A. A. 1967, AZh, 44, 1016

Fekel, F. C., Joyce, R. R., Hinkle, K. H., \& Skrutskie, M. F. 2000, AJ, 119, 1375 
Fernandez-Castro, T., Cassatella, A., Gimenez, A., \& Viotti, R. 1988, ApJ, 324, 1016

Fernandez-Castro, T., Gonzalez-Riestra, R., Cassatella, A., Taylor, A., \& Seaquist, E. R. 1995, ApJ, 442, 366

Formiggini, L., \& Leibowitz, E. M. 1994, A\&A, 292, 534

Fujimoto, M. Y. 1982, ApJ, 257, 767

Johnson, H. L. 1966, ARA\&A, 4, 163

Kilpio, E. Yu., Bisikalo, D. V., Boyarchuk, A. A., \& Kuznetsov, O. A. 2004, in ASP Conf. Ser., The Astrophysics of Cataclysmic Variables and Related Objects, ed. J. M. Hameury, \& J. P. Lasota

Mihailov, A. A. 1973, Kurs astrofiziki i zvezdnoj astronomii (Moscow: Nauka), in russian

Mikolajewska, J., \& Kenyon, S. J. 1992, MNRAS, 256, 177

Mikolajewska, J., \& Kenyon, S. J. 1996, AJ, 112, 1659

Mürset, U., \& Schmid, H. M. 1999, A\&AS, 137, 473

Nussbaumer, H., \& Vogel, M. 1989, A\&A, 213, 137

Osterbrock, D. E. 1974, Astrophysics of Gaseous Nebulae (San Francisco: W.H. Freeman and Company), 73
Schmid, H. M., \& Schild, H. 1997, A\&A, 327, 219

Seaton, M. J. 1979, MNRAS, 187, 73P

Skopal, A. 2003, A\&A, 401, L17

Skopal, A., Chochol, D., Pribulla, T., \& Vanko, M. 2000, IBVS, 5005

Skopal, A., Vanko, M., Pribulla, T., et al. 2002, Contrib. Astron. Obs. Skalnate Pleso, 32, 1

Skopal, A., Pribulla, T., Vanko, M., et al. 2004, Contrib. Astron. Obs. Skalnate Pleso, 34, 45

Sokoloski, J. L., \& Bildsten, L. 1999, ApJ, 517, 919

Swings, P., \& Struve, O. 1941, ApJ, 93, 356

Tomov, N. A., Taranova, O. G., \& Tomova, M. T. 2003a, A\&A, 401, 669 (Paper I)

Tomov, N. A., Tomova, M. T., \& Zamanov, R. K. 2003b, in Symbiotic Stars Probing Stellar Evolution, ed. R. L. M. Corradi, J. Mikolajewska, \& T. J. Mahoney, ASP Conf. Ser., 303, 254

Tomov, N. A., Tomova, M. T., \& Taranova, O. G. 2004, in ASP Conf. Ser., The Astrophysics of Cataclysmic Variables and Related Objects, ed. J. M. Hameury, \& J. P. Lasota 